\title{
SEROMA FORMATION IN CANCER BREAST SURGERY
}

\author{
P. S. Sitaram ${ }^{1}$, R. Hemanthi ${ }^{2}$
}

\section{HOW TO CITE THIS ARTICLE:}

P. S. Sitaram, R. Hemanthi. "Seroma Formation in Cancer Breast Surgery". Journal of Evolution of Medical and Dental Sciences 2015; Vol. 4, Issue 10, February 02; Page: 1681-1688, DOI: 10.14260/jemds/2015/237

ABSTRACT: Post-operative breast surgery for malignancy has a common side effect of seromas, whose formation and drainage requires a special attention to prevent as much as possible and as early as possible to avoid morbidity. How best we can prevent the dead space is the problem. In this study a review of various methods adopted all over the world is reviewed including early discharge of the patient with drain. It is always preferable to remove the drain when the collection of serum is less than $25 \mathrm{ml}$ in 24 hours the chance of re accumulation of fluid is less likely. INTRODUCTION: Carcinoma breast has remained the second leading cause of cancer death among women worldwide over the past three decades ${ }^{[1]}$ and contributes significantly to cancer surgical load. Surgical treatment for breast cancer includes breast conservation therapy and mastectomy with or without axillary dissection depending on disease stage. Seroma formation is the most frequent postoperative Side effect seen after mastectomy and axillary surgery with an incidence of 3\% to 85\%. OBSERVATIONS: TYPE OF SURGERY: Surgical treatment for breast cancer has undergone a paradigm shift from Halstead's radical mastectomy to breast conservation. It has been demonstrated that radical mastectomy increases seroma formation compared with that of simple mastectomy, but the association is inconclusive when radical mastectomy is compared with modified radical mastectomy (MRM). SURGICAL DEVICES: Various electro-mechanical devices are used during surgery to reduce blood loss and operating time. These include electrocautery, laser scalpel, argon diathermy, ultrasonic scalpel, ultrasonic scissors, and vessel sealing systems. All of these devices have been investigated in an effort to reduce seroma formation. Randomized trials have shown that the use of electrocautery for dissecting flaps is significantly associated with increased seroma formation when compared to that of scalpel dissection. However, no individual study has shown a significant effect on seroma formation with or without the use of a laser scalpel, argon diathermy, or an ultrasonic scalpel. Ultrasonic scissors resulted in reduced seroma formation in a randomized controlled trial comparing level I and II axillary dissection using either ultrasound scissors or surgical scissors with ligation. MANAGEMENT OF DEAD SPACE: MECHANICAL DEVICES: Different techniques have been employed to obliterate the dead space (under flaps and the axilla) to reduce seroma formation. CHEMICAL: Fibrin glue.light activated fibrin sealant, and transdermal photo-polymerized adhesive reduce seroma formation after mastectomy in animal models. Use of a fibrinolysis inhibitor was based on the hypothesis that fibrinolytic activity in serum and lymph might contribute to fluid accumulation. SHOULDER MOVEMENT: Shoulder dysfunction is a common complication of mastectomy,and it is necessary to mobilize the shoulder early to prevent this complication. It was thought that early shoulder mobilization led to increased seroma formation and this hypothesis was supported by a systematic review of 12 randomized controlled trials (RCTs) of which six were included in a metaanalysis. CONCLUSIONS: Seroma formation is a side effect of M.R.M rather than a complication Physical closure of the dead space appears to reduce seroma rate, but studies have failed to address the issues of cosmesis, and shoulder movement restrictions and these add to the operating time. and mechanical pressure do not reduce seroma drainage. Drains should be used, but the number of drain 
tubes or a low pressure system does not decrease the formation of seroma. However, low vacuum drains in the axilla result in less seroma formation, earlier drain removal and earlier discharge. High pressure vacuum drains appear safe and acceptable to discharge many patients early with drains in situ, if adequate patient counseling and nursing support are provided. Seroma formation can be safely considered on an outpatient basis by multiple percutaneous aspirations.

KEYWORDS: Breast cancer, Seroma.

INTRODUCTION: Carcinoma breast has remained the second leading cause of cancer death among women worldwide over the past three decades ${ }^{[1]}$ and contributes significantly to cancer surgical load. Surgical treatment for breast cancer includes breast conservation therapy and mastectomy with or without axillary dissection depending on disease stage. Seroma formation is the most frequent postoperative Side effect seen after mastectomy and axillary surgery with an incidence of $3 \%$ to 85\%. ${ }^{[2]}$ Associated morbidity in the form of prolonged drainage is not only troublesome to the patient but can also significantly impact treatment by delaying adjuvant therapy and increasing the risk for infection. ${ }^{[2]}$ A reoperation may be necessary for cases of longstanding persistent seroma.[3] This review updates the various factors thought to contribute to seroma formation and the probable interventions that may be of help to reduce incidence.

PATHOPHYSIOLOGY: Seroma after breast surgery is defined as a serous fluid collection that develops under the skin flaps or in the axillary dead space following mastectomy and/or axillary dissection. The origin of seroma remains unclear but several risk factors and predictors are age, breast size, extent of dissection comorbid conditions, presence and number of malignant nodes in the axilla, previous surgical biopsy, and use of heparin or tamoxifen.[4-6] It has been hypothesized that seromas form as an exudate from an acute inflammatory reaction following surgical trauma[6] to increase serous fluid collection in response to increased fibrinolytic activity in serum and lymph.[7] Low fibrinogen levels in seromas compared with those in plasma during the postoperative period[8] support the hypothesis that seroma most likely originates from lymph. ${ }^{[9]}$ Seroma formation is influenced by an array of surgical techniques and devices;[10-13] thus, leading to varying incidence of seroma in different studies.

\section{OBSERVATIONS:}

TYPE OF SURGERY: Surgical treatment for breast cancer has undergone a paradigm shift from Halstead's radical mastectomy to breast conservation. It has been demonstrated that radical mastectomy increases seroma formation compared with that of simple mastectomy,[14,15] but the association is inconclusive when radical mastectomy is compared with modified radical mastectomy (MRM).[14] Conversely patients undergoing MRM have a significant increased incidence of seroma formation when compared to those who have breast conservation surgery.[16] Preservation or removal of the pectoral fascia has no effect on the incidence of seroma.[17] It has also been observed that immediate breast reconstruction following MRM decreases seroma formation when compared to a delayed procedure.[18] The number of removed lymph nodes probably does not influence seroma formation. ${ }^{[19,20]}$ A randomized controlled trial by Purushotham et al.[21] demonstrated that sentinel lymph node biopsy is associated with significantly less seroma formation than that of conventional axillary dissection. 
SURGICAL DEVICES: Various electro-mechanical devices are used during surgery to reduce blood loss and operating time. These include electrocautery, laser scalpel, argon diathermy, ultrasonic scalpel, ultrasonic scissors, and vessel sealing systems. All of these devices have been investigated in an effort to reduce seroma formation. Randomized trials have shown that the use of electrocautery for dissecting flaps is significantly associated with increased seroma formation when compared to that of scalpel dissection. ${ }^{[12,22]}$ However, no individual study has shown a significant effect on seroma formation with or without the use of a laser scalpel,[23] argon diathermy,[13] or an ultrasonic scalpel.[24] Ultrasonic scissors resulted in reduced seroma formation in a randomized controlled trial comparing level I and II axillary dissection using either ultrasound scissors or surgical scissors with ligation. ${ }^{[25]}$ An Italian group compared the bipolar vessel sealing system with conventional surgical dissection and found no difference in the duration of the surgical procedure, total drainage fluid volume, drainage duration, or postoperative adverse events between the groups in a randomized trial.[26] Interestingly, a significant increase in seroma was observed in the vessel sealing system group. However other studies have reported improved results with use of a vessel sealing system. One of these was a prospective study, ${ }^{[27]}$ in which the results were compared with historical data, and decreased drainage duration and hospital stay were observed. Another retrospective study concluded that the drainage duration is significantly shorter with the use of a vessel sealing system but not the cost of treatment. The benefit in terms of fluid loss also remains to be demonstrated.[28] The differences in outcomes probably reflect differences in study methods; thus, further randomized trials with larger sample sizes are required.

\section{MANAGEMENT OF DEAD SPACE:}

MECHANICAL DEVICES: Different techniques have been employed to obliterate the dead space (under flaps and the axilla) to reduce seroma formation. Halsted first advocated creating a short superior flap and suturing it with interrupted silk to the fascia below the first rib and skin grafting the remaining part of the defect.[29] In 1951, Orr ${ }^{[30]}$ used tension sutures tied over rubber tubing bolsters to tack flaps to the chest wall. In 1953, Keyes et al.[31] used through and through sutures to attach the skin flaps to the chest wall. Besides these techniques, suturing of flaps with subcutaneous tissue,[32] avoiding use of axillary drains following breast conservation therapy,[33] and obliterating axillary dead space by muscle approximation have all been tried for reducing seroma formation. Coveney et al. compared suturing skin flaps to underlying muscle with conventional skin closure and observed a lower incidence of seroma formation in the flap suture group, although flap suturing did add to total operating time. A recent randomized study compared a combination of skin flap suturing, ligation of lymphatics and obliteration of axillary dead space to conventional skin closure after mastectomy. As a result, the incidence of seroma formation decreased to $2 \%$ with the combination of techniques. Although effective, the authors stated that it was impossible to determine which of the three techniques, or any combination, actually produced the observed effect. Mechanical pressure has also been applied to obliterate dead space following surgery[34]. The use of a pressure garment does not reduce postoperative drainage and has low tolerance and a higher complication rate.

CHEMICAL: Fibrin glue. Light activated fibrin sealant, and transdermal photo-polymerized adhesive reduce seroma formation after mastectomy in animal models. Use of a fibrinolysis inhibitor was based on the hypothesis that fibrinolytic activity in serum and lymph might contribute to fluid 
accumulation. Sanders et al. reported that fibrinogen and thrombin concentrations in the fibrin sealant are proportional to the reduction in seroma formation. However, no significant difference in the incidence of seroma formation occurred with the use of fibrin glue in human studies.In contrast, Vaxman et al. demonstrated in a randomized trial that use of fibrin glue actually increases seroma formation rate. The advantage of using fibrin glue comes from three other studies that demonstrated significantly reduced total seroma drainage, early drain removal, and reduced hospital stay. Most of these studies had a limitation of a relatively small sample size. A reduction in postoperative drainage and hospital stay were observed following use of fibrin glue, but it did not affect delayed seroma formation. However, the use of fibrin glue or peri-operative and postoperative administration of a fibrinolysis inhibitor does not reduce seroma formation.

SHOULDER MOVEMENT: Shoulder dysfunction is a common complication of mastectomy, and it is necessary to mobilize the shoulder early to prevent this complication. It was thought that early shoulder mobilization led to increased seroma formation and this hypothesis was supported by a systematic review of 12 randomized controlled trials (RCTs) of which six were included in a metaanalysis. The study showed that a delayed shoulder exercise program reduces seroma formation (odds ratio, $0.4 ; 95 \%$ confidence interval, $0.2-0.5 ; \mathrm{p}=0.00001$ ) but no differences were found for drainage volume or hospital stay. Conversely, a number of RCTs have demonstrated no difference in seroma formation between early (within 1-2 days postoperatively) or late (by 5-7 days postoperatively) shoulder movement. Temporary immobilization of the shoulder using a collar and cuff ${ }^{20]}$ or sling has been attempted with an aim to reduce seroma formation but was not found to be beneficial. Thus, the present evidence does not support shoulder immobilization.

Another parallel issue is whether active shoulder mobilization through physiotherapy has any effect on seroma formation. A number of reports comparing delayed physiotherapy, even until removal of the drain showed less total wound drainage, shorter drainage period, and a shorter hospital stay without any difference in the functional range of movement in the longer term. Thus delayed physiotherapy may reduce seroma formation at the expense of mild short-term shoulder dysfunction but without long term restriction of movement.

DRAINS-CLOSED/OPEN: The use of drains has been a common practice to obliterate the dead space created after surgery. The use of closed suction drainage in patients who underwent mastectomy accelerates wound healing and is also associated with a lower incidence of wound infection, necrosis, and breakdown. The choice of the number of drain tubes used has been studied. use of multiple drains does not confer any significant advantage on either the amount or duration of seroma drainage.

Studies comparing the intensity of negative drain suction have shown mixed results. In a study of 46 patients who underwent mastectomy, randomized between high vacuum drain and low vacuum drain, seroma drainage and postoperative hospital stay was longer in the low vacuum system group than that in the high vacuum system possibly because the high vacuum drain led to more efficient flap approximation to the chest wall. No significant difference was observed in the volume of axillary fluid produced, drainage duration or wound complication rates between the two groups. High vacuum drains had a higher incidence of vacuum loss but a lower incidence of leakage around the 


\section{REVIEW ARTICLE}

drain. Thus, no strong evidence is available to recommend high or low pressure suction to reduce seroma formation.

EARLY DISCHARGE WITH DRAIN IN SITU: Patients can be safely discharged with drains in situ with adequate patient education and coordination of inpatient and outpatient facilities. Acceptance rates for early discharge with drains in situ vary between $24 \%$ and $41 \%$, Interestingly, patients who choose early discharge tend to be significantly younger, are living with another adult, and are more likely to have had breast-conserving surgery Concerns expressed in the early discharge group of patients include personal care, bed posture, dressing themselves, fatigue, loneliness, pain, and worries about the wound and the arm. Despite these factors, studies have shown that patient acceptance of early discharge with drains in situ remains good. However, some reports have expressed mixed results and no compelling evidence points to a uniform reduction in seroma rate following early discharge with a drain in situ, although discharge within a day of surgery has been fraught with a higher rate of seroma formation.

EARLY DRAIN REMOVAL: It is common practice to remove drains when drainage decreases to a minimal volume (20-50 $\mathrm{mL}$ ) in the preceding 24 hours to minimize seroma formation. It has been shown that 48 hours after surgery, as much as $74 \%$ of the total volume of seroma has been drained. It has also been observed that drains may be safely removed after axillary dissection, if the total drainage during the first 3 days is less than $250 \mathrm{~mL}$. No significant difference was observed with respect to drainage volume at the time of drain removal, subsequent mean number of aspirations, and time to resolution of seromas. More seroma fluid was collected in the group whose drain was left in situ longer, but no difference in the volume, number, or duration of percutaneous aspirations was observed once the drain was removed. No significant difference in drainage at 48 hours and no significant difference in seroma frequency were observed. Liu and McFadden. Only a 2\% seroma rate was observed, as 49 out of the 50 patients had no symptomatic seroma. Thus, there appears to be good evidence in favor of early drain removal.

NO WOUND DRAINAGE: When drainage and no drainage were compared in patients who underwent lumpectomy and axillary dissection,[19] short duration closed suction drainage appeared advantageous for decreasing the incidence and degree of seroma formation and did not seem to delay early hospital discharge. The results showed a significantly higher rate of seroma formation in the undrained group (45\% vs. 10\%). All seromas resolved clinically within 1 month or within 4 months on ultrasonographic examination. More aspirations in the no-drain group were required (50\%) compared to that in the drain group (8.3\%), but no difference was observed in the complication rate and the pain rating was significantly less in the no-drain group.

PATIENT FACTORS: Although a number of surgical technique-related factors have been described to play a role in seroma formation, most patient and tumor-related factors have been shown consistently to have no significant association with seroma formation. A number of studies have attempted to associate patient and tumour characteristics to postoperative seroma formation. Body weight ${ }^{[2]}$ and body mass index are associated with increased seroma formation, whereas no consistent association has been found between seroma formation and hormone receptor status,[26] 
axillary nodal status, lymph node positivity[18,20] or disease stage and grade.[18,26] Similarly, no consistent association has been found between seroma formation and the presence of anemia, smoking, diabetes mellitus, or breast size.[14] Tumor size and location, histological type, site of the disease and specimen weight are not associated with increased seroma formation.[26]

CONCLUSIONS: Seroma formation is a side effect of M.R.M rather than a complication but can delay patient recovery and cause unpleasant symptoms. Patient and tumor-related factors have no significant bearing on seroma formation except possibly body weight and body mass index, which seem to be directly proportional to seroma formation. Physical closure of the dead space appears to reduce seroma rate, but studies have failed to address the issues of cosmesis, and shoulder movement restrictions and these add to the operating time. And mechanical pressure do not reduce seroma drainage. Shoulder immobilization is of no advantage to the patient, but it appears that delaying shoulder physiotherapy reduces drainage. Drains should be used, but the number of drain tubes or a low pressure system does not decrease the formation of seroma. However, low vacuum drains in the axilla result in less seroma formation, earlier drain removal and earlier discharge. High pressure vacuum drains in the axilla may promote increased drainage due to flap irregularity and poor flap adherence. High pressure vacuum drains appear safe and acceptable to discharge many patients early with drains in situ, if adequate patient counseling and nursing support are provided. Seroma formation can be safely considered on an outpatient basis by multiple percutaneous aspirations.

\section{REFERENCES:}

1. Jemal A, Siegel R, Ward E, Hao Y, Xu J, Murray T, et al. Cancer statistics, 2008. CA Cancer J Clin. 2008; 58: 71-96. [PubMed]

2. Kumar S, Lal B, Misra MC. Post-mastectomy seroma: a new look into the aetiology of an old problem. J R Coll Surg Edinb. 1995; 40: 292-294. [PubMed]

3. Stanczyk M, Grala B, Zwierowicz T, Maruszynski M. Surgical resection for persistent seroma, following modified radical mastectomy. World J Surg Oncol. 2007; 5: 104. [PMC free article] [PubMed].

4. Bryant $\mathrm{M}$, Baum M. Postoperative seroma following mastectomy and axillary dissection. Br J Surg. 1987; 74: 1187. [PubMed].

5. Willett WC, Rockhill B, Hankinson SE, Hunter DJ, Colditz GA. Epidemiology and nongenetic causes of breast cancer. In: Harris JR, Lippman ME, Morrow M, Osborne CK, editors. Diseases of the Breast. $2^{\text {nd }}$ ed. Philadelphia: Lippincott Williams \& Wilkins; 2000. pp. 175-220.

6. Watt-Boolsen S, Nielsen VB, Jensen J, Bak S. Postmastectomy seroma. A study of the nature and origin of seroma after mastectomy. Dan Med Bull. 1989; 36: 487-489. [PubMed]

7. Oertli D, Laffer U, Haberthuer F, Kreuter U, Harder F. Perioperative and postoperative tranexamic acid reduces the local wound complication rate after surgery for breast cancer. Br J Surg. 1994; 81: 856-859. [PubMed]

8. Bonnema J, van Geel AN, Wiggers T, Ligtenstein DA. Perioperative and postoperative tranexamic acid reduces the local wound complication rate after surgery for breast cancer. Br J Surg. 1994; 81: 1693. [PubMed] 
9. Bonnema J, Ligtenstein DA, Wiggers T, van Geel AN. The composition of serous fluid after axillary dissection. Eur J Surg. 1999; 165: 9-13. [PubMed]

10. Tejler G, Aspegren K. Complications and hospital stay after surgery for breast cancer: a prospective study of 385 patients. Br J Surg. 1985; 72: 542-544. [PubMed]

11. Keogha GW, Doughty JC, McArdle CS, Cookea TG. Seroma formation related to electrocautery in breast surgery: a prospective randomized trial. Breast. 1998; 7: 39-41.

12. Porter KA, O'Connor S, Rimm E, Lopez M. Electrocautery as a factor in seroma formation following mastectomy. Am J Surg. 1998; 176: 8-11. [PubMed]

13. Kerin MJ, O'Hanlon DM, Kenny P, Kent PJ, Given HF. Argon-enhanced cutting and coagulation confers advantages over conventional electrocautery for mastectomy. Eur J Surg Oncol. 1996; 22: 571-573. [PubMed]

14. Say CC, Donegan W. A biostatistical evaluation of complications from mastectomy. Surg Gynecol Obstet. 1974; 138: 370-376. [PubMed]

15. Aitken DR, Hunsaker R, James AG. Prevention of seromas following mastectomy and axillary dissection. Surg Gynecol Obstet. 1984; 158: 327-330. [PubMed]

16. Gonzalez EA, Saltzstein EC, Riedner CS, Nelson BK. Seroma formation following breast cancer surgery. Breast J. 2003; 9: 385-388. [PubMed]

17. Dalberg K, Johansson H, Signomklao T, Rutqvist LE, Bergkvist L, Frisell J, et al. A randomised study of axillary drainage and pectoral fascia preservation after mastectomy for breast cancer. Eur J Surg Oncol. 2004; 30: 602-609. [PubMed]

18. Woodworth PA, McBoyle MF, Helmer SD, Beamer RL. Seroma formation after breast cancer surgery: incidence and predicting factors. Am Surg. 2000; 66: 444-450. [PubMed]

19. Somers RG, Jablon LK, Kaplan MJ, Sandler GL, Rosenblatt NK. The use of closed suction drainage after lumpectomy and axillary node dissection for breast cancer. A prospective randomized trial. Ann Surg. 1992; 215: 146-149. [PMC free article] [PubMed]

20. Browse DJ, Goble D, Jones PA. Axillary node clearance: who wants to immobilize the shoulder? Eur J Surg Oncol. 1996; 22: 569-570. [PubMed]

21. Purushotham AD, Upponi S, Klevesath MB, Bobrow L, Millar K, Myles JP, et al. Morbidity after sentinel lymph node biopsy in primary breast cancer: results from a randomized controlled trial. J Clin Oncol. 2005; 23: 4312-4321. [PubMed]

22. Hashemi E, Kaviani A, Najafi M, Ebrahimi M, Hooshmand H, Montazeri A. Seroma formation after surgery for breast cancer. World J Surg Oncol. 2004; 2: 44. [PMC free article][PubMed]

23. Wyman A, Rogers K. Randomized trial of laser scalpel for modified radical mastectomy.Br J Surg. 1993; 80: 871-873. [PubMed]

24. Galatius H, Okholm M, Hoffmann J. Mastectomy using ultrasonic dissection: effect on seroma formation. Breast. 2003; 12: 338-341. [PubMed]

25. Lumachi F, Brandes AA, Burelli P, Basso SM, Iacobone M, Ermani M. Seroma prevention following axillary dissection in patients with breast cancer by using ultrasound scissors: a prospective clinical study. Eur J Surg Oncol. 2004; 30: 526-530. [PubMed]

26. Antonio M, Pietra T, Domenico L, Massimo D, Ignazio R, Antonio N, et al. Does LigaSure(TM) reduce fluid drainage in axillary dissection? A randomised prospective clinical trial. Ecancermedicalscience. 2007; 1: 61. [PMC free article] [PubMed] 
27. Manouras A, Markogiannakis H, Genetzakis M, Filippakis GM, Lagoudianakis EE, Kafiri G, et al. Modified radical mastectomy with axillary dissection using the electrothermal bipolar vessel sealing system. Arch Surg. 2008; 143: 575-580. [PubMed]

28. Magri K, Bertrand V, Sautière JL, Pivot X, Riethmuller D, Maillet R, et al. Cost effectiveness of Ligasure Precise versus surgical clips during axillary nodal dissection for breast cancer. J Gynecol Obstet Biol Reprod (Paris) 2006; 35: 341-347. [PubMed]

29. Almond LM, Khodaverdi L, Kumar B, Coveney EC. Flap anchoring following primary breast cancer surgery facilitates early hospital discharge and reduces costs. Breast Care (Basel) 2010; 5: 97-101. [PMC free article] [PubMed]

30. Orr TG., Jr An incision and method of wound closure for radical mastectomy. Ann Surg.1951; 133: 565-566. [PMC free article] [PubMed]

31. Keyes EL, Hawk BO, Sherwin CS. Basting the axillary flap for wounds of radical mastectomy. AMA Arch Surg. 1953; 66: 446-451. [PubMed]

32. Larsen BB, Hugan C., Jr Fixation of skin flaps in radical mastectomy by subcutaneous sutures: observations. AMA Arch Surg. 1955; 71: 419-423. [PubMed]

33. Schuijtvlot M, Sahu AK, Cawthorn SJ. A prospective audit of the use of a buttress suture to reduce seroma formation following axillary node dissection without drains. Breast. 2002; 11: 94-96. [PubMed]

34. Hamy A, Bouchot-Hermouet FB, Bouchot O, Drianno JC, Guillard Y. Wadding of the axilla in the conservative treatment of cancer of the breast. Prevention of lymphocele. J Chir (Paris) 1990; 127: 99-102. [PubMed]

\section{AUTHORS:}

1. P.S. Sitaram

2. R. Hemanthi

\section{PARTICULARS OF CONTRIBUTORS:}

1. Assistant Professor, Department of Surgery, RMC/GGH, Kakinada.

2. Assistant Professor, Department of Surgery, RMC/GGH, Kakinada.

\section{NAME ADDRESS EMAIL ID OF THE CORRESPONDING AUTHOR:}

Dr. P. S. Sitaram,

\# 8-7-2, Sri Satya Sai Hospital, Gandhinagar, Kakinada-533004.

E-mail: drsaipindi@gmail.com

Date of Submission: 09/01/2015.

Date of Peer Review: 10/01/2015.

Date of Acceptance: 22/01/2015.

Date of Publishing: 31/01/2015. 